\title{
Limit on Lorentz and CPT Violation of the Neutron Using a Two-Species Noble-Gas Maser
}

\author{
D. Bear, R. E. Stoner, and R. L. Walsworth \\ Harvard-Smithsonian Center for Astrophysics, Cambridge, Massachusetts 02138 \\ V. Alan Kostelecký and Charles D. Lane \\ Physics Department, Indiana University, Bloomington, Indiana 47405
}

(Received 14 July 2000)

\begin{abstract}
A search for sidereal variations in the frequency difference between co-located ${ }^{129} \mathrm{Xe}$ and ${ }^{3} \mathrm{He}$ Zeeman masers sets the most stringent limit to date on leading-order Lorentz and $C P T$ violation involving the neutron, consistent with no effect at the level of $10^{-31} \mathrm{GeV}$.

PACS numbers: 06.30.Ft, 11.30.Cp, 11.30.Er, 84.40.Ik
\end{abstract}

Lorentz symmetry is a fundamental feature of modern descriptions of nature, including both the standard model of particle physics and general relativity. However, these realistic theories are believed to be the low-energy limit of a single fundamental theory at the Planck scale. Even if the underlying theory is Lorentz invariant, spontaneous symmetry breaking might result in small apparent violations of Lorentz invariance at an observable level. Experimental investigations of the validity of Lorentz symmetry therefore provide valuable tests of the framework of modern theoretical physics.

Clock-comparison experiments [1-6] serve as sensitive probes of rotation invariance and hence of Lorentz symmetry, essentially by bounding the frequency variation of a clock as its orientation changes. In practice, the most precise limits are obtained by comparing the frequencies of two different co-located clocks as they rotate with the Earth. Typically, the clocks are electromagnetic signals emitted or absorbed on hyperfine or Zeeman transitions.

Here, we report on a search for sidereal variations in the frequency of co-located ${ }^{129} \mathrm{Xe}$ and ${ }^{3} \mathrm{He}$ masers, both operating on nuclear spin- $1 / 2$ Zeeman transitions. In the context of a general standard-model extension allowing for the possibility of Lorentz and $C P T$ violation $[7,8]$, the ${ }^{129} \mathrm{Xe} /{ }^{3} \mathrm{He}$-maser experiment sets the most stringent limit to date on leading-order Lorentz and $C P T$ violation of the neutron: about $10^{-31} \mathrm{GeV}$, or more than 6 times better than the best previous measurements [9].

The standard-model extension used to interpret this experiment emerges from any underlying theory that reduces at low energy to the standard model and contains spontaneous Lorentz violation [10]. For example, this might occur in string theory [11]. The standard-model extension maintains theoretically desirable properties of the usual standard model [8]. Its formulation at the level of the known elementary particles is a key feature enabling quantitative comparison of a wide array of tests of Lorentz and $C P T$ symmetry. In this context, theoretical studies have been performed to investigate the sensitivity of clockcomparison experiments [9], tests of QED in Penning traps [12], experiments with a spin-polarized torsion pendulum
[13], hydrogen-antihydrogen spectroscopy [14], studies of photon birefringence in the vacuum $[8,15,16]$, experiments with muons [17], measurements of neutral-meson oscillations [7,18], studies of the baryon asymmetry [19], and cosmic-ray observations [20].

In the context of the standard-model extension, the most sensitive prior clock-comparison experiment is the ${ }^{199} \mathrm{Hg} /{ }^{133} \mathrm{Cs}$ comparison of Hunter, Lamoreaux et al. $[6,9]$. Recent experimental work motivated by the standard-model extension includes Penning-trap tests by Gabrielse et al. on the antiproton and the $\mathrm{H}^{-}$ion [21] and by Dehmelt et al. on the electron and positron $[22,23]$. A reanalysis by Adelberger, Gundlach, Heckel, and co-workers of existing data from a spin-polarized torsion-pendulum experiment $[24,25]$ sets the most stringent bound to date on Lorentz and CPT violation of the electron, at about $10^{-28} \mathrm{GeV}$ [26]. A recent Lorentzsymmetry test using hydrogen masers searched for hydrogen Zeeman-frequency sidereal variations, placing a bound on Lorentz violation at the level of $10^{-27} \mathrm{GeV}$ [27]. Together with the results of Ref. [26], this implies an improved clean limit of $10^{-27} \mathrm{GeV}$ on Lorentz-violating couplings involving the proton. Also, the $\mathrm{KTeV}$ experiment at Fermilab and the OPAL and DELPHI collaborations at CERN have constrained possible Lorentz- and $C P T$-violating effects in the $K$ and $B_{d}$ systems [28,29].

The design and operation of the two-species ${ }^{129} \mathrm{Xe} /{ }^{3} \mathrm{He}$ maser has been discussed in recent publications [30,31]. Here, we give a brief review. The two-species maser contains dense co-located ensembles of ${ }^{3} \mathrm{He}$ and ${ }^{129} \mathrm{Xe}$ atoms. Each ensemble performs an active maser oscillation on its nuclear spin-1/2 Zeeman transition at approximately $4.9 \mathrm{kHz}$ for ${ }^{3} \mathrm{He}$ and $1.7 \mathrm{kHz}$ for ${ }^{129} \mathrm{Xe}$ in a static magnetic field of $1.5 \mathrm{G}$. This two-species maser operation can be maintained indefinitely. The population inversions for the two maser ensembles are created by spin-exchange collisions between the noble-gas atoms and optically pumped $\mathrm{Rb}$ vapor $[32,33]$. The ${ }^{129} \mathrm{Xe} /{ }^{3} \mathrm{He}$ maser has two glass chambers, one acting as the spin exchange "pump bulb" and the other serving as the "maser bulb." This two chamber configuration permits the combination of physical 
conditions necessary for a high flux of spin-polarized noble-gas atoms into the maser bulb, while also maintaining ${ }^{3} \mathrm{He}$ - and ${ }^{129} \mathrm{Xe}$-maser oscillations with good frequency stability: stability of about $100 \mathrm{nHz}$ is typical for measurement intervals larger than about an hour [31]. Either noble-gas species can serve as a precision magnetometer to stabilize the system's static magnetic field, while the other species is employed as a sensitive probe for Lorentz- and $C P T$-violating interactions.

We used the ${ }^{129} \mathrm{Xe} /{ }^{3} \mathrm{He}$ maser to search for a Lorentzviolation signature by monitoring the relative phases and Larmor frequencies of the co-located ${ }^{3} \mathrm{He}$ and ${ }^{129} \mathrm{Xe}$ masers as the laboratory reference frame rotated with respect to the distant stars. The system was operated with the ${ }^{129} \mathrm{Xe}$ maser as the comagnetometer, the ${ }^{3} \mathrm{He}$ maser free running, and the quantization axis directed east-west in the Earth's reference frame. To leading order, the Lorentz-violating couplings of either the ${ }^{3} \mathrm{He}$ or ${ }^{129} \mathrm{Xe}$ nucleus can be treated as those of a single ${ }^{1} S_{1 / 2}$ valence neutron in a Schmidt model [9]. Thus, the Lorentz-violating Zeeman frequency shifts $\left(\delta \nu_{J}^{\text {Lorentz }}\right)$ for ${ }^{3} \mathrm{He}$ and ${ }^{129} \mathrm{Xe}$ are the same size and sign. The magnitude of the sidereal variation of the freerunning ${ }^{3} \mathrm{He}$-maser frequency $\left(\delta \nu_{J}\right)$, accounting for the effect of the ${ }^{129} \mathrm{Xe}$-maser comagnetometer, is given by

$$
\delta \nu_{J}=\delta \nu_{J}^{\text {Lorentz }}\left|1-\gamma_{\mathrm{He}} / \gamma_{\mathrm{Xe}}\right| \simeq 1.75 \delta \nu_{J}^{\text {Lorentz }} .
$$

Here, $\gamma_{\mathrm{He}} / \gamma_{\mathrm{Xe}} \simeq 2.75$ is the ratio of ${ }^{3} \mathrm{He}$ and ${ }^{129} \mathrm{Xe}$ gyromagnetic ratios. The subscript $J=X, Y$ indicates components in the sidereal reference frame that are orthogonal to the Earth's axis of rotation. Hence, the sidereal variation of the free-running ${ }^{3} \mathrm{He}$-maser frequency observed in the laboratory frame takes the form

$$
\delta \nu_{\mathrm{He}}=\delta \nu_{X} \cos \left(\Omega_{s} t\right)+\delta \nu_{Y} \sin \left(\Omega_{s} t\right),
$$

where $\Omega_{s}$ is the angular frequency of the sidereal day [34]. The time $t$ was measured in seconds from the beginning of the sidereal day in Cambridge, Massachusetts (longitude $\left.-71.11^{\circ}\right)$

Data collection and analysis were performed as follows. The ${ }^{129} \mathrm{Xe}$ - and ${ }^{3} \mathrm{He}$-maser signals from an inductive pickup coil were buffered, amplified, and sent to a pair of digital lock-in detectors. Typical raw-signal levels were about 3 to $5 \mu \mathrm{V}$. All reference signals used in the experiment were derived from the same hydrogen-maser clock, thus eliminating concerns about unmeasurable electronic phase shifts between the reference oscillators. The hydrogen maser operated on the standard hyperfine clock transition, and thus had no leading-order sensitivity to Lorentz and $C P T$ violation $[9,14]$. Active feedback to the solenoid's magnetic field locked the phase of the ${ }^{129} \mathrm{Xe}$ maser to that of a $1.7 \mathrm{kHz}$ reference signal and thereby isolated the experiment from common-mode systematic effects (such as stray magnetic-field fluctuations) that would otherwise shift the frequencies of the noble-gas masers in proportion to the ratio of their magnetic moments. When phase locked, the short- and long- term frequency stability [31] of the ${ }^{129} \mathrm{Xe}$ maser was several orders of magnitude better than that of the free-running ${ }^{3} \mathrm{He}$ maser, so the ${ }^{129} \mathrm{Xe}$ Zeeman frequency was treated as constant in the data analysis.

The phase and amplitude of both maser signals were recorded at four-second intervals by the lock-in amplifiers and downloaded for analysis every $23.93 \mathrm{~h}$. A onesidereal-day run thus contained approximately 21540 evenly spaced measurements of the relative phases of the two masers. The values of the two coefficients $\delta \nu_{X}, \delta \nu_{Y}$ were computed, providing a measure of potential Lorentz violation for that day's run. Seven additional diagnostic signals were recorded, including the temperatures of the pump bulb, maser bulb, and external resonator; an optical monitor of the Rb magnetization in the pump bulb; the broadband power emitted by the optical-pumping laser-diode array; the ambient room temperature; and the east-west component of the ambient magnetic field. Control loops stabilized the system temperatures to about $10 \mathrm{mK}$. Two additional control loops stabilized the $\mathrm{Rb}$ magnetization in the pump bulb, and thus the amplitudes of the noble-gas masers, to about one part in $10^{3}$. The broadband power of the optical-pumping laser-diode array (LDA) was locked by picking off a fraction of the output light, observing it with a photodetector after passing it through a chopper, and feeding back a control signal to the LDA temperature. The Rb magnetization could then be stabilized by slightly dithering the $\mathrm{Rb}$ polarization with weak rf applied near the Rb Zeeman resonance in the pump bulb, observing the resultant modulation of resonant LDA light passing through the pump bulb, and sending a control signal to a liquid-crystal variable retarder to adjust the incident-light polarization. With the two control loops engaged, there was no discernible drift of the $\mathrm{Rb}$ magnetization over time scales of many days.

Small noble-gas polarization-induced frequency shifts were the dominant source of instability (i.e., phase drift) in the free-running ${ }^{3} \mathrm{He}$ maser. For a typical one-day run, the linear-correlation coefficient between ${ }^{3} \mathrm{He}$ phase data and the integrated amplitude of either maser was in the range $0.95-0.99$. We admitted terms to our dataanalysis model to account for this polarization-induced phase drift. The effect of potential Lorentz-violating couplings on the evolution of the ${ }^{3} \mathrm{He}$ phase was expressed in terms of the coefficients $\delta \nu_{J}$ via integration of Eq. (2), and initial reduction of each one-day run was performed using the minimal fit model

$$
\begin{aligned}
\delta \phi_{\mathrm{He}}= & \phi_{0}+2 \pi \nu_{0} t \\
& +2 \pi \Omega_{s}^{-1}\left[\delta \nu_{X} \sin \left(\Omega_{s} t\right)-\delta \nu_{Y} \cos \left(\Omega_{s} t\right)\right],
\end{aligned}
$$

where the coefficients $\phi_{0}$ and $\nu_{0}$ account for absolute phase and frequency offsets between the ${ }^{3} \mathrm{He}$ maser and the ultrastable reference oscillator. The reduced $\chi^{2}$ statistic for this fit model was determined, and then additional terms corresponding to quadratic and maser amplitudecorrelated phase drift were incorporated into the model 
if they significantly improved the reduced $\chi^{2}$. The coefficients $\delta \nu_{X}$ and $\delta \nu_{Y}$ for each one-day run were extracted using a linear least-squares routine on the best-fitting model for that day, which contained at most seven free parameters and thus at least $(21500-7)$ degrees of freedom. See Fig. 1 for an example of the residuals from one day's data. As a final check, a faux Lorentz-violating effect of known phase and amplitude was added to the raw data and the analysis was repeated. Data reduction for a given sidereal day was considered successful if the synthetic physics was recovered and there was no change in the covariance matrix generated by the fitting routine.

Data for this experiment were acquired with three different maser cells over a period of 30 days in April 1999 (cell S3), 24 days in September 1999 (cell E9), and 60 days in February-May 2000 (cell SE3, runs 1 and 2). A total of 90 usable sidereal-day values of $\delta \nu_{X}, \delta \nu_{Y}$ were obtained. The main magnetic field of the apparatus was reversed about every 10 days to help distinguish possible Lorentz-violating effects from diurnal systematic variations. Field reversal and subsequent reequilibration of the masers required approximately $24 \mathrm{~h}$.

Systematic effects resulting from possible diurnally varying ambient magnetic fields would not average away with field reversals. Thus, the effectiveness of the ${ }^{129} \mathrm{Xe}$ comagnetometer at eliminating such effects was carefully assessed before beginning data acquisition. Since the two maser ensembles do not have perfect spatial overlap, penetration of external magnetic fields through the nested magnetic shields and into the interaction region could induce small frequency shifts in the free-running ${ }^{3} \mathrm{He}$ maser despite the presence of the ${ }^{129} \mathrm{Xe}$ comagnetometer. Large coils $\left(\sim 2.4 \mathrm{~m}\right.$ diameter) surrounding the ${ }^{129} \mathrm{Xe} /{ }^{3} \mathrm{He}-$ maser apparatus were used to switch on and off $0.5 \mathrm{G}$ external magnetic fields in the north-south and east-west directions. A bound on the ratio $\left|\delta \nu_{\mathrm{He}} / \delta B_{\text {external }}\right|$ was obtained. The drifts in the ambient magnetic field near

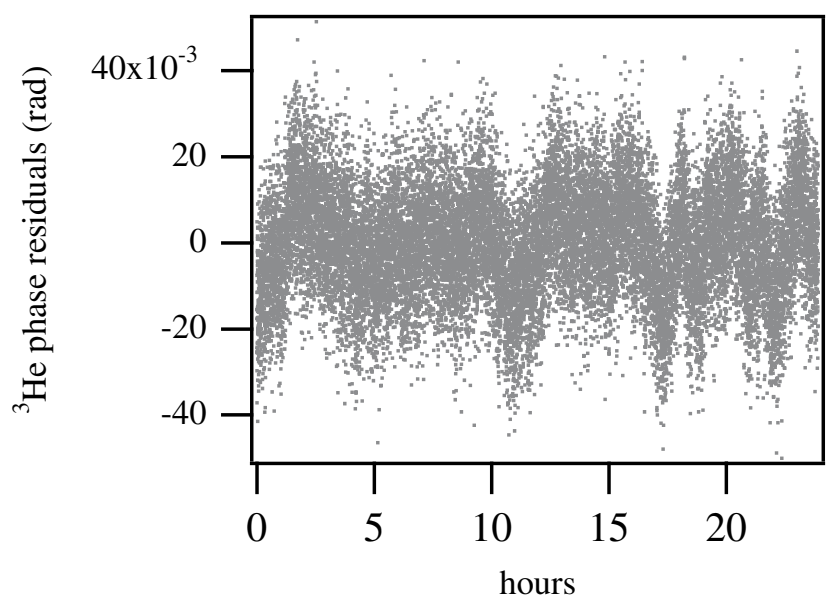

FIG. 1. Typical residuals for the ${ }^{3} \mathrm{He}$ phase data from one sidereal day, calculated using the fit model given in Eq. (3). the apparatus were measured to be about $0.2 \mathrm{mG}$ over a typical 24-h period, resulting in a worst-case shift on the free-running ${ }^{3} \mathrm{He}$ maser of less than $8 \mathrm{nHz}$, well below the present sensitivity of the experiment to Lorentz and $C P T$ violation. It should be noted that the relative phase between the solar and the sidereal day evolved about $2 \pi$ radians over the course of the experiment (April 1999 to May 2000). Hence, diurnal systematic effects from any source would tend to be reduced by averaging results from all measurement sets.

For each cell, the data for the east and west magneticfield orientations were analyzed separately to determine mean values and standard errors for $\delta \nu_{J}$, yielding the results in Table I. As an example, Fig. 2 shows the single-day values of $\delta \nu_{X}$ obtained in the first run with cell SE3 in the east field orientation (SE3 E1). The measured values of $\delta \nu_{J}$ exhibited no significant dependence on the direction of the solenoidal magnetic field in the laboratory frame.

The total weighted means and uncertainties for $\delta \nu_{X}$ and $\delta \nu_{Y}$ were formed from all data sets. These values were then used to extract the rms magnitude of the correction to the ${ }^{3} \mathrm{He}$ Zeeman frequency due to Lorentz-violating couplings in the $X Y$ plane, $R \equiv \sqrt{\delta \nu_{X}^{2}+\delta \nu_{Y}^{2}}$, yielding $53 \pm 45 \mathrm{nHz}(1-\sigma$ level). This result is consistent with no Lorentz- and $C P T$-violating effects, given reasonable assumptions about the probability distribution for $R$ [35].

In terms of the standard-model extension, Eq. (1) becomes [9]

$$
2 \pi\left|\delta \nu_{J}\right|=\left|-3.5 \tilde{b}_{J}^{n}+0.012 \tilde{d}_{J}^{n}-0.012 \tilde{g}_{D, J}^{n}\right| .
$$

Here, $\tilde{b}_{J}^{n}, \tilde{d}_{J}^{n}$, and $\tilde{g}_{D, J}^{n}$ are small parameters characterizing the strength of Lorentz-violating couplings of the neutron to possible background tensor fields that may arise from spontaneous symmetry breaking in a fundamental theory. The couplings associated with $\tilde{b}_{J}^{n}$ and $\tilde{g}_{D, J}^{n}$ also violate $C P T$. All three parameters are linear combinations of more basic quantities in the relativistic Lagrangian of the standard-model extension [9].

The coefficients in Eq. (4) include the effect of the ${ }^{129} \mathrm{Xe}$ comagnetometer and indicate that the ${ }^{129} \mathrm{Xe} /{ }^{3} \mathrm{He}-$ maser experiment is most sensitive to the Lorentz- and

TABLE I. Means and standard errors for $\delta \nu_{X}$ and $\delta \nu_{Y}$. Results are displayed for each of the three cells (S3, E9, and SE3) with both east (E) and west (W) orientations of the magnetic field. Two runs were performed for cell SE3.

\begin{tabular}{crr}
\hline \hline Cell & $\delta \nu_{X}(\mathrm{nHz})$ & $\delta \nu_{Y}(\mathrm{nHz})$ \\
\hline S3 E & $95 \pm 118$ & $197 \pm 114$ \\
S3 W & $-43 \pm 138$ & $88 \pm 148$ \\
E9 E & $-86 \pm 234$ & $-194 \pm 207$ \\
E9 W & $-206 \pm 186$ & $-60 \pm 134$ \\
SE3 E1 & $100 \pm 148$ & $9 \pm 141$ \\
SE3 W1 & $-1 \pm 88$ & $62 \pm 109$ \\
SE3 E2 & $-2 \pm 180$ & $68 \pm 107$ \\
SE3 W2 & $-35 \pm 118$ & $197 \pm 120$ \\
\hline \hline
\end{tabular}




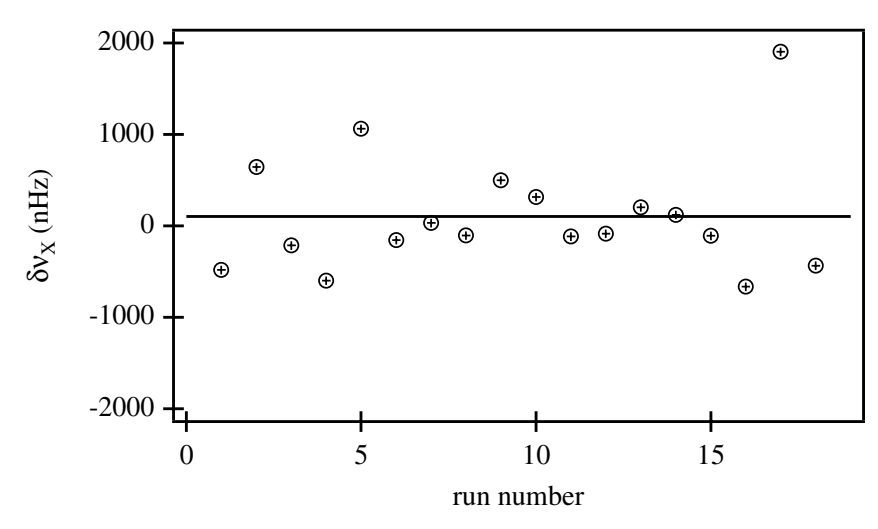

FIG. 2. Values of the Lorentz-violating parameter $\delta \nu_{X}$ obtained with cell SE3 in the E1 orientation. The horizontal line indicates the mean value for that data set.

$C P T$-violating couplings associated with $\tilde{b}_{J}^{n}$. Under the assumption of negligible contribution from the $\tilde{d}_{J}^{n}$ and $\tilde{g}_{D, J}^{n}$ terms [9], the above experimental result for $R$ corresponds to a value for $\tilde{b}_{\perp}^{n} \equiv \sqrt{\left(\tilde{b}_{X}^{n}\right)^{2}+\left(\tilde{b}_{Y}^{n}\right)^{2}}=$ $(4.0 \pm 3.3) \times 10^{-31} \mathrm{GeV}$. This is the most stringent limit to date on possible Lorentz- and $C P T$-violating couplings involving the neutron and is more than 6 times better than the best previous measurements [9].

We are planning improved Lorentz and $C P T$ tests using noble-gas masers. Upgrading laser and temperature control and acquiring a larger data set could better the present constraint from the ${ }^{129} \mathrm{Xe} /{ }^{3} \mathrm{He}$ system by up to an order of magnitude. Also, a new two-species Zeeman maser using ${ }^{3} \mathrm{He}$ and ${ }^{21} \mathrm{Ne}$ might provide even greater improvements to constraints on neutron parameters $[9,36]$.

David Phillips, Mark Rosenberry, Federico Cané, Timothy Chupp, Robert Vessot, and Edward Mattison helped greatly with this project. Support was provided by NASA Grant No. NAG8-1434, ONR Grant No. N00014-99-1-0501, and DOE Grant No. DE-FG0291ER40661.

[1] V. W. Hughes, H. G. Robinson, and V. Beltran-Lopez, Phys. Rev. Lett. 4, 342 (1960).

[2] R. W. P. Drever, Philos. Mag. 6, 683 (1961).

[3] J. D. Prestage et al., Phys. Rev. Lett. 54, 2387 (1985).

[4] S. K. Lamoreaux et al., Phys. Rev. A 39, 1082 (1989).

[5] T.E. Chupp et al., Phys. Rev. Lett. 63, 1541 (1989).

[6] C. J. Berglund et al., Phys. Rev. Lett. 75, 1879 (1995); L. R. Hunter et al., in CPT and Lorentz Symmetry, edited by V. A. Kostelecký (World Scientific, Singapore, 1999).

[7] V. A. Kostelecký and R. Potting, Phys. Rev. D 51, 3923 (1995); Gamma Ray-Neutrino Cosmology and Planck Scale Physics, edited by D. B. Cline (World Scientific, Singapore, 1993), hep-th/9211116.

[8] D. Colladay and V. A. Kostelecký, Phys. Rev. D 55, 6760 (1997); 58, 116002 (1998).

[9] V. A. Kostelecký and C. D. Lane, Phys. Rev. D 60, 116010 (1999); J. Math. Phys. 40, 6425 (1999).
[10] V. A. Kostelecký and S. Samuel, Phys. Rev. Lett. 63, 224 (1989); Phys. Rev. D 40, 1886 (1989); Phys. Rev. Lett. 66, 1811 (1991).

[11] V. A. Kostelecký and S. Samuel, Phys. Rev. D 39, 683 (1989); V. A. Kostelecký and R. Potting, Nucl. Phys. B359, 545 (1991); Phys. Lett. B 381, 89 (1996); hep-th/0008252; V. A. Kostelecký, M. Perry, and R. Potting, Phys. Rev. Lett. 84, 4541 (2000).

[12] R. Bluhm, V. A. Kostelecký, and N. Russell, Phys. Rev. Lett. 79, 1432 (1997); Phys. Rev. D 57, 3932 (1998).

[13] R. Bluhm and V. A. Kostelecký, Phys. Rev. Lett. 84, 1381 (2000).

[14] R. Bluhm, V. A. Kostelecký, and N. Russell, Phys. Rev. Lett. 82, 2254 (1999).

[15] S. M. Carroll, G. B. Field, and R. Jackiw, Phys. Rev. D 41, 1231 (1990).

[16] R. Jackiw and V. A. Kostelecký, Phys. Rev. Lett. 82, 3572 (1999). See also M. Pérez-Victoria, Phys. Rev. Lett. 83, 2518 (1999); J. M. Chung, Phys. Lett. B 461, 138 (1999).

[17] R. Bluhm, V. A. Kostelecký, and C. D. Lane, Phys. Rev. Lett. 84, 1098 (2000).

[18] D. Colladay and V. A. Kostelecký, Phys. Lett. B 344, 259 (1995); Phys. Rev. D 52, 6224 (1995); V. A. Kostelecký and R. Van Kooten, Phys. Rev. D 54, 5585 (1996); V. A. Kostelecký, Phys. Rev. Lett. 80, 1818 (1998); Phys. Rev. D 61, 16002 (2000).

[19] O. Bertolami et al., Phys. Lett. B 395, 178 (1997).

[20] S. Coleman and S.L. Glashow, Phys. Rev. D 59, 116008 (1999).

[21] G. Gabrielse et al., Phys. Rev. Lett. 82, 3198 (1999).

[22] R. K. Mittleman et al., Phys. Rev. Lett. 83, 2116 (1999).

[23] H. G. Dehmelt et al., Phys. Rev. Lett. 83, 4694 (1999).

[24] E. G. Adelberger et al., in Physics Beyond the Standard Model, edited by P. Herczeg et al. (World Scientific, Singapore, 1999).

[25] M. G. Harris, Ph.D. thesis, University of Washington, 1998.

[26] B. Heckel, in Proceedings of the International Conference on Orbis Scientiae 1999, Fort Lauderdale, Florida (Plenum, New York, 2000).

[27] D. F. Phillips et al., physics/0008230 (to be published).

[28] Y.B. Hsiung, in Proceedings of the KAON 99 Conference, Chicago, 1999 (University of Chicago Press, Chicago, 2000).

[29] OPAL Collaboration, R. Ackerstaff et al., Z. Phys. C 76, 401 (1997); DELPHI Collaboration, M. Feindt et al., Report No. DELPHI 97-98 CONF 80, 1997.

[30] R. E. Stoner et al., Phys. Rev. Lett. 77, 3971 (1996).

[31] D. Bear et al., Phys. Rev. A 57, 5006 (1998).

[32] T. E. Chupp et al., Phys. Rev. A 38, 3998 (1988).

[33] G. D. Cates et al., Phys. Rev. A 45, 4631 (1992).

[34] One sidereal day is approximately one solar day scaled by $(1-1 / 364.24)$, or about $23.93 \mathrm{~h}$.

[35] The probability distribution for $R$ is formed by multiplying the distributions for $\delta \nu_{X}$ and $\delta \nu_{Y}$, converting to polar coordinates, and integrating over the polar angle. In the case where $\delta \nu_{X}$ and $\delta \nu_{Y}$ have zero mean value and the same variance $\sigma$, the probability distribution takes the form $P(R)=\sigma^{-2} R \exp \left(-r^{2} / 2 \sigma^{2}\right)$, with the most probable value of $R$ occurring at $R=\sigma$.

[36] R. E. Stoner, in CPT and Lorentz Symmetry (Ref. [6]). 